\title{
Correction to: A susceptibility-weighted imaging qualitative score of the motor cortex may be a useful tool for distinguishing clinical phenotypes in amyotrophic lateral sclerosis
}

\author{
Giorgio Conte ${ }^{1}$ (D) Sara Sbaraini ${ }^{2}$ (D) $\cdot$ Claudia Morelli ${ }^{3}$ (D) Silvia Casale ${ }^{1} \cdot$ Luca Caschera $^{1} \cdot$ Valeria Elisa Contarino $^{1}$. \\ Elisa Scola ${ }^{1}$. Claudia Cinnante ${ }^{1}$ - Francesca Trogu ${ }^{3,4} \cdot$ Fabio Triulzi $^{1,4} \cdot$ Vincenzo Silani $^{3,4}$ (ID
}

Published online: 27 November 2020

(C) European Society of Radiology 2020

\section{Correction to: European Radiology}

https://doi.org/10.1007/s00330-020-07239-0

The original version of this article, published on 04 September 2020, unfortunately contained a mistake. The following correction has therefore been made in the original: The first and last names of the authors were interchanged. The corrected author list is given above. The original article has been corrected.

Publisher's note Springer Nature remains neutral with regard to jurisdictional claims in published maps and institutional affiliations.

The online version of the original article can be found at https://doi.org/ $10.1007 / \mathrm{s} 00330-020-07239-0$

Sara Sbaraini

sara.sbaraini@gmail.com

1 Neuroradiology Unit, Fondazione IRCCS Ca' Granda Ospedale Maggiore Policlinico, via Francesco Sforza 35, Milan, Italy

2 Neuroradiology Unit, Department of Radiology, ASST Santi Paolo e Carlo, San Carlo Borromeo Hospital, via Pio II n. 3, Milan, Italy

3 Department of Neurology-Stroke Unit and Laboratory of Neuroscience, Istituto Auxologico Italiano IRCCS, piazzale Brescia 20, Milan, Italy

4 Department of Pathophysiology and Transplantation, Università degli Studi di Milano, via Festa del Perdono 7, Milan, Italy 